\title{
Comparative metabolome analysis of serum changes in sheep under overgrazing or light grazing conditions
}

\author{
Jize Zhang ${ }^{1}$, Yang Gao², Huiqin Guo ${ }^{3}$, Yong Ding ${ }^{1}$ and Weibo Ren ${ }^{4 *}$
}

\begin{abstract}
Background: Overgrazing is a primary contributor to severe reduction in forage quality and production in Inner Mongolia, leading to extensive ecosystem degradation, sheep health impairment and growth performance reduction. Further studies to identify serum biomarkers that reflect changes in sheep health and nutritional status following overgrazing would be beneficial. We hereby hypothesize that reduced sheep growth performance under overgrazing conditions would be associated with metabolic and immune response alterations. This study used an untargeted metabolomics analysis by conducting ultra-high-performance liquid chromatography combined with quadrupole time-of-flight mass spectrometry (UHPLC-Q-TOF/MS) of sheep serum under overgrazing and light grazing conditions to identify metabolic disruptions in response to overgrazing.

Results: The sheep body weight gains as well as serum biochemical variables associated with immune responses and nutritional metabolism (immunoglobulin G, albumin, glucose, and nonesterified fatty acids) were significantly decreased with overgrazing compared with light grazing condition. In contrast, other serum parameters such as alanine and aspartate aminotransferase, alkaline phosphatase, total bilirubin, blood urea nitrogen, and interleukin-8 were markedly higher in the overgrazing group. Principal component analysis discriminated the metabolomes of the light grazing from the overgrazing group. Multivariate and univariate analyses revealed changes in the serum concentrations of 15 metabolites ( 9 metabolites exhibited a marked increase, whereas 6 metabolites showed a significant decrease) in the overgrazing group. Major changes of fatty acid oxidation, bile acid biosynthesis, and purine and protein metabolism were observed.

Conclusions: These findings offer metabolic evidence for putative biomarkers for overgrazing-induced changes in serum metabolism. Target-identification of these particular metabolites may potentially increase our knowledge of the molecular mechanisms of altered immune responses, nutritional metabolism, and reduced sheep growth performance under overgrazing conditions.
\end{abstract}

Keywords: Overgrazing, Light grazing, Serum metabolome, Sheep

\section{Background}

Overgrazing is the most severe threat to the typical steppe of Inner Mongolia. In recent decades, the grassland ecosystem as well as mutton and milk production have been extensively damaged due to overgrazing. Gains in the stocking rate have resulted in the accumulation of volatile organic compounds and morphological alterations in grassland plants [1]. Multiyear or single

\footnotetext{
* Correspondence: rppcaucau@163.com

${ }^{4}$ School of Ecology and Environment, Inner Mongolia University, Hohhot 010021, Inner Mongolia, China

Full list of author information is available at the end of the article
}

year research studies have revealed that overgrazing induces a significant decline in sheep (15-mo-old female) live body weight gain per hectare or individual in grazing season (June to September) [2,3]. The reason for that is because overgrazing has severely damaged this natural grassland, which degrades the normal morphology of forages (lower forage production and imbalanced nutrients composition) resulting in reduction of the individual animal growth performance. With the growing emphasis on grazing animal welfare, increasing attention has been placed on the potential for the health status and nutrient metabolism of grazing sheep to be altered 
by increasd grazing intensity, especially under overgrazing conditions [4]. In response to overgrazing induced undernutrition, the long-term response of animal was characterized by nutrients mobilization (energy, protein and so on), which triggered tissue masses decrease, especially fat and muscle tissues. This may be a primary reason for the reduced growth performance observed in overgrazing sheep. Proteomics data obtained from our former study also demonstrated that overgrazing may trigger a shift in energy resources from carbohydrates to proteins due to the shortage supply of forage and imbalanced nutrients composition [4]. Furthermore, various proteins associated with immune response were downregulated in the hepatic proteome of sheep in response to overgrazing [4]. A number of metabolites related to sheep immune responses and nutritional metabolism may be altered by overgrazing induced forage degradation and lower animal growth performance. However, few quantitative data are available on the metabolic profile alterations of sheep under overgrazing conditions.

Recently, metabolomics has become a powerful high-throughput bioanalytical method for detecting important metabolite biomarkers in ruminants in response to different diets or environmental stresses [5-7]. In a previous metabolomics study, analysis of serum and urine samples collected from sheep after $12 \mathrm{~h}$ and $48 \mathrm{~h}$ of road transport by nuclear magnetic resonance (NMR) spectroscopy revealed peroxisomal fatty acid oxidation as a metabolic response to transport-induced stress [8]. The metabolomic profile of rumen fluid in dairy cows fed a high-concentrate diet demonstrated altered concentrations of ruminal metabolites (bacterial degradation products, toxic compounds and amino acids) as well as an altered metabolic pattern [9]. Liquid chromatography/mass spectrometry (LC/MS) analysis of serum samples of lactating dairy cows under controlled heat stress revealed 13 potential biomarkers involved in carbohydrate, amino acid, lipid, or gut microbiome-derived metabolism, indicating that heat stress affected the metabolic pathways in lactating dairy cows [10]. To date, most metabolomics studies on ruminants focus on the zero-grazing system. However, this advanced bioanalytical method is still not widely used to evaluate alterations of the health status and nutritional metabolism of animals under overgrazing conditions.

Therefore, the goals of the present study were to identify and characterize the potential biomarkers in sheep serum that are associated with the consequences of overgrazing. The findings of this study provide critical information that may be utilized to improve the production of grazing animals and for the sustainable development of the Inner Mongolia steppe.

\section{Results}

Herbage nutritional contents, sheep growth performance and serum biochemical parameters

For herbage nutritional contents, crude protein $(\mathrm{CP})$ and acid detergent lignin (ADL) were significantly higher in the overgrazing (OG) group than in the light grazing (LG) group $(P<0.05)$, nitrogen free extract (NFE) was significantly lower in the OG group than in the LG group $(P<0.05)$, and there was not significant difference in gross energy between the two groups (see Additional file 1). By the end of the grazing experiment, the body weight gain of sheep in the OG group was $12.2 \mathrm{~kg}$, while that in the LG group was $14.9 \mathrm{~kg}(P<0.05)$ (Table 1). For serum biochemical parameters, alanine aminotransferase (ALT), aspartate aminotransferase (AST), alkaline phosphatase (ALP), total bilirubin (TBIL), serum blood urea nitrogen (BUN) and interleukin-8 (IL-8) were significantly higher in the OG group than in the LG group $(P<0.05)$; albumin (ALB), glucose (GLU), nonesterified fatty acids (NEFAs) and immunoglobulin $\mathrm{G}$ ( $\operatorname{IgG}$ ) were significantly lower in the OG group than in the LG group $(P<0.05)$ (Table 2$)$.

\section{Plasma metabolic profiling and different serum metabolites between the $L G$ and $O G$ groups}

Figure 1 showed the distribution of metabolic profiles in the quality control $(\mathrm{QC})$ samples from Pareto-scaled principal component analysis (PCA). Clear clustering of QCs in PCA space was shown for both the electrospray ionization (ESI) $(+)$ and ESI (-) modes. The consistently repeated QC injections as well as reliable data quality among samples indicated the excellent repeatability, performance and stability of the sample treatment procedure.

To assess differences in global serum metabolite fingerprints between LG and OG sheep, the separation in both ion modes between the LG and OG sheep by the unsupervised PCA method was evaluated. As shown in Fig. 2, the two groups exhibited minimal but not significant differences in trend in the 2D PCA plots. We thus conducted further multivariate statistical analysis to establish the relationship between the two groups.

Supervised orthogonal partial least-squares discriminant analysis (OPLS-DA) is a powerful method with which to discriminate ion peaks that contribute to the

Table 1 Effect of overgrazing on the growth performance of sheep

\begin{tabular}{|c|c|c|c|c|c|c|}
\hline \multirow[t]{2}{*}{ Group } & \multicolumn{4}{|c|}{ Body weight (kg) } & \multirow{2}{*}{$\begin{array}{l}\text { Body } \\
\text { weight } \\
\text { gain (kg) }\end{array}$} & \multirow[b]{2}{*}{ SEM } \\
\hline & Initial & SEM & Final & SEM & & \\
\hline G & $33.1 \pm 1.1$ & 0.3 & $48.1 \pm 3.0^{a}$ & 0.9 & $14.9 \pm 3.3^{\mathrm{a}}$ & 0.9 \\
\hline 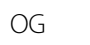 & $33.0 \pm 4.7$ & 1.4 & $45.4 \pm 5.9^{b}$ & 1.7 & $12.2 \pm 2.5^{b}$ & 0.7 \\
\hline
\end{tabular}

Numbers are expressed as the means \pm SDs. $(n=12)$. The means within a column with different superscript letters are significantly different at $P<0.05$ 
Table 2 The effect of overgrazing on sheep serum biochemical variables

\begin{tabular}{|c|c|c|c|}
\hline Item (serum activity/concentration) & LG & OG & SEM \\
\hline$\overline{\mathrm{ALT}(\mathrm{IU} / \mathrm{L})}$ & $28.51 \pm 9.75^{b}$ & $39.30 \pm 7.32^{a}$ & 2.27 \\
\hline AST (IU/L) & $125.25 \pm 13.03^{b}$ & $148.26 \pm 15.30^{a}$ & 3.57 \\
\hline $\operatorname{ALP}(U / L)$ & $203.43 \pm 11.76^{b}$ & $270.71 \pm 26.35^{a}$ & 3.89 \\
\hline $\mathrm{ALB}(\mathrm{g} / \mathrm{L})$ & $32.36 \pm 4.78^{\mathrm{a}}$ & $26.73 \pm 4.21^{\mathrm{b}}$ & 0.89 \\
\hline TBIL (mmol/L) & $4.13 \pm 0.66^{b}$ & $6.26 \pm 1.37^{\mathrm{a}}$ & 0.21 \\
\hline BUN (mmol/L) & $8.75 \pm 0.38^{b}$ & $11.20 \pm 1.55^{\mathrm{a}}$ & 0.26 \\
\hline $\mathrm{GLU}(\mathrm{mmol} / \mathrm{L})$ & $5.95 \pm 1.26^{\mathrm{a}}$ & $4.38 \pm 0.87^{b}$ & 0.30 \\
\hline NEFAs (mmol/L) & $0.62 \pm 0.05^{\mathrm{a}}$ & $0.37 \pm 0.08^{b}$ & 0.02 \\
\hline IL-8 (pg/mL) & $0.48 \pm 0.07^{b}$ & $0.75 \pm 0.11^{\mathrm{a}}$ & 0.02 \\
\hline $\lg G(g / L)$ & $17.12 \pm 2.57^{\mathrm{a}}$ & $13.79 \pm 1.83^{b}$ & 0.45 \\
\hline
\end{tabular}

LG light grazing, OG overgrazing

ALT alanine aminotransferase, AST aspartate aminotransferase, ALP alkaline phosphatase, ALB albumin, TBIL total bilirubin, BUN blood urea nitrogen, GLU glucose, NEFAs nonesterified fatty acid, IL interleukin, IgG immunoglobulin G

Numbers are expressed as the means \pm SDs. $(n=12)$. The means within a row with different superscript letters differ significantly at $P<0.05$

classification of samples and remove noncorrelated variations contained within spectra. Thus, OPLS-DA was conducted to confirm the separation and detect potential biomarkers between the LG and OG groups. Clear separation between the LG and OG groups based on the first two components was detected based on the OPLSDA plot using the positive ion mode $\left(R^{2} Y=0.984\right.$, $\left.\mathrm{Q}^{2} \mathrm{Y}=0.751\right)$ (Fig. 3a) and the negative ion mode $\left(\mathrm{R}^{2} \mathrm{Y}=\right.$ $0.990, Q^{2} Y=0.869$ ) (Fig. 3b), with $R^{2} Y$ indicating the goodness of the fit, with $\mathrm{Q}^{2} \mathrm{Y}$ indicating the model's prediction ability. The models showed good sample classification into two groups, which was suggestive that the model was the high reliability and had predictive power. In both the PCA or OPLS-DA plot, the separation between the LG and OG groups was superior in both positive and negative modes, which indicated that sheep under OG conditions exhibited some alterations in the levels of a few metabolites.
Based on statistical analysis and the variable importance in the projection (VIP) value obtained from the OPLS-DA, 15 significantly different serum metabolites were selected as potential biomarkers related to OG (Table 3). Among the different alterations observed, the concentration of 6 metabolites was remarkably higher and of 9 metabolites was significantly lower in the OG group than in the LG group.

To further evaluate alternations in the serum metabolome caused by OG, we conducted hierarchical clustering analysis using the degree of similarity in metabolite abundance profiles (Fig. 4a and b). Metabolites with identical abundance patterns were clustered together. The heatmap dendrogram revealed the tighter clustering of the OG-induced metabolites, including their separation from the LG group.

The identified metabolites influencing OG play a major role in particular metabolic pathways. Therefore,
A

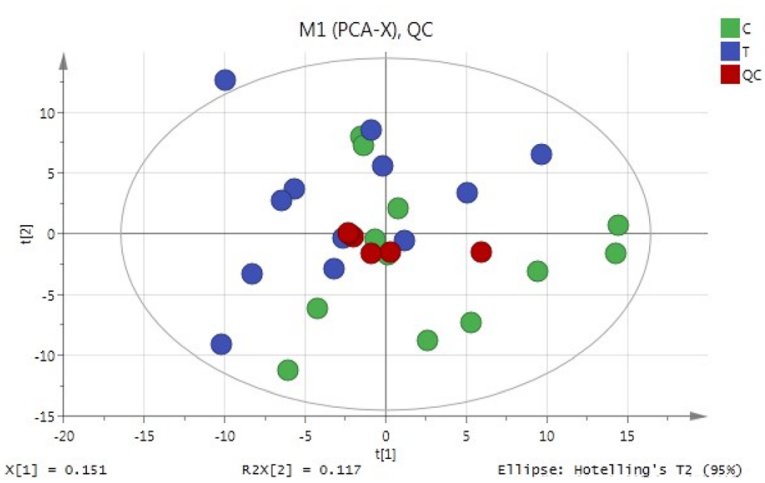

B

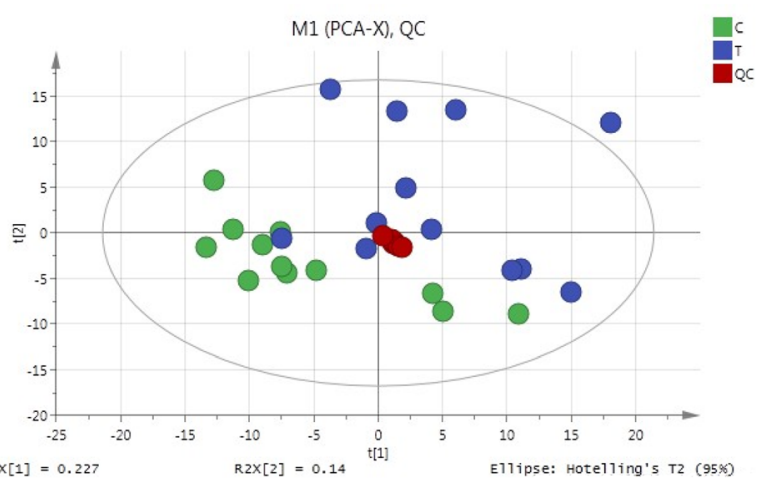

Fig. 1 PCA scores in ESI positive mode (a) and ESI negative mode (b) based on the ultra-high-performance liquid chromatography combined with quadrupole time-of-flight mass spectrometry (UHPLC-Q-TOF/MS) data of the serum samples. Green dots: serum samples of sheep under LG conditions; blue dots: serum samples of sheep under OG conditions; red dots: QC samples 


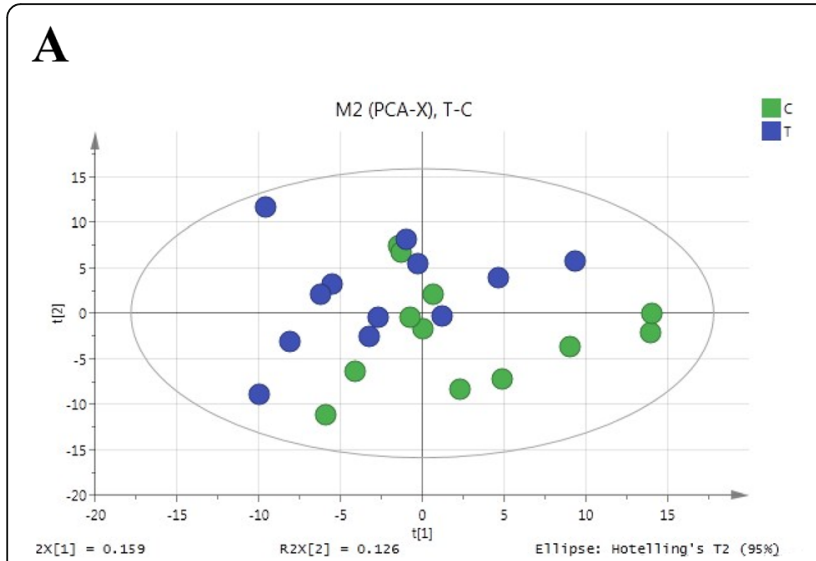

B

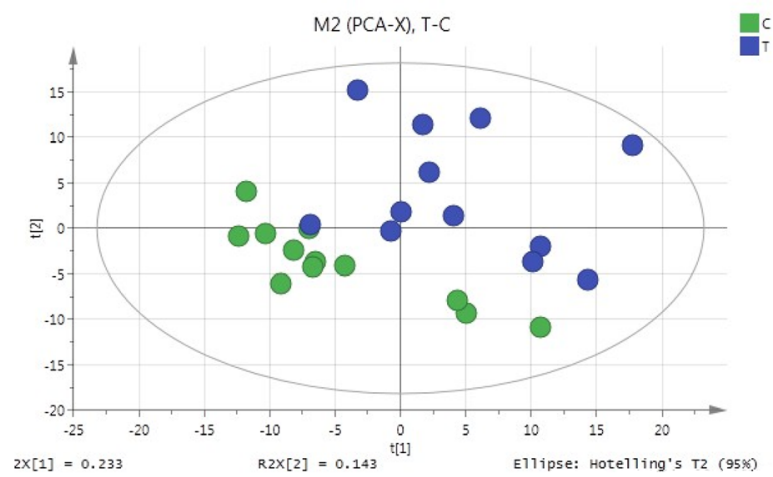

Fig. 2 PCA score map derived from UHPLC-Q-TOF/MS spectra for LG sheep (green dots) and OG sheep (blue dots) in the positive mode (a) and negative mode (b)

to determine possible pathways influenced by OG, the metabolites were assessed using the Kyoto Encyclopedia of Genes and Genomes (KEGG) pathway database (http://www.genome.jp/kegg/) (Table 3). Metabolomics analysis indicated that the variable effects of OG on various metabolic pathways in serum could be detected to follow an untargeted manner. These findings revealed that the potential biomarkers contribute to various processes, including fatty acid metabolism, bile acid synthesis, and purine and protein metabolism. (Table 3). Various metabolic pathways were thus induced by alterations in OG-induced sheep physiological function and might be closely associated with these changes.

\section{Discussion}

The present study is to explore the biofluids of sheep under OG conditions to identify metabolic profiles that could be link to animal growth performance. Our previously published data demonstrated that various hepatic responses in sheep were triggered by OG (proteomic profiles) [4]. However, the serum metabolic changes of sheep under OG conditions were still largely unknown. In this study, LC/MS-based metabolomics was used to compare the metabolisms of sheep under LG and OG conditions, which will provide a comprehensive overview of the metabolic profile to help understand the molecular mechanisms of reduced animal growth performance in response to OG. Our results revealed that sheep growth performance as well as the serum metabolome was significantly influenced by OG. Furthermore, nutrients composition of pasture was also changed greatly under OG conditions, especially CP, ADL and NFE. The reason for this may be because the composition of plant community was altered by OG [11]. However, this speculation is still required to be demonstrated in future studies, which is not the focus of the present research.

Stearoylcarnitine and L-palmitoylcarnitine are longchain acylcarnitines, which are markers of lipid
A

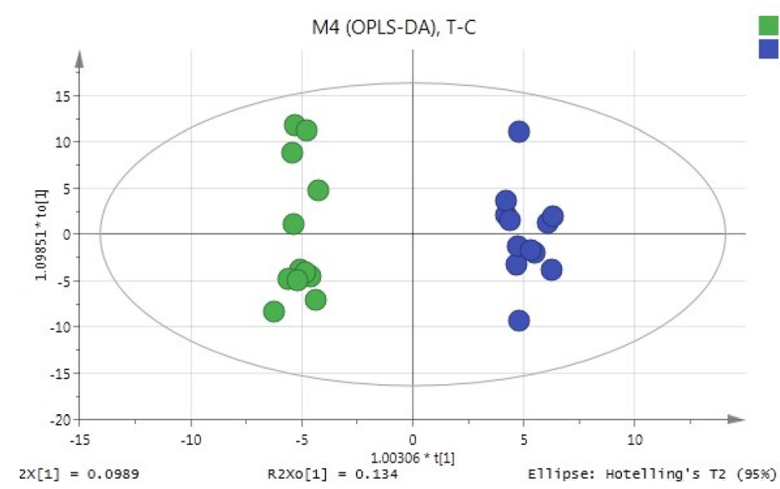

B

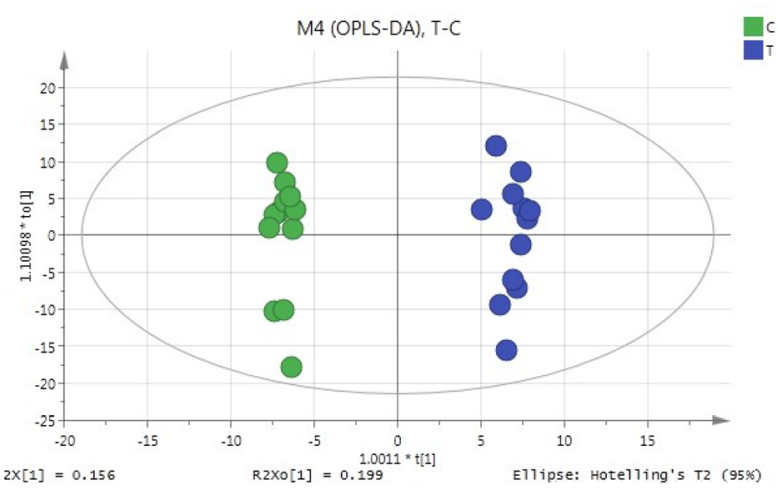

Fig. 3 OPLS-DA score map derived from UHPLC-Q-TOF/MS spectra for LG sheep (green dots) and OG sheep (blue dots) in the positive mode (a) and negative mode (b) 
Table 3 List of significantly changed compounds in sheep from overgrazing group and light grazing group

\begin{tabular}{|c|c|c|c|c|c|c|c|c|}
\hline $\begin{array}{l}\text { lonization } \\
\text { mode }\end{array}$ & Metabolites & Adduct & VIP & $\begin{array}{l}\text { Fold } \\
\text { change }\end{array}$ & $P$ value & $\begin{array}{l}\text { Retention } \\
\text { time (S) }\end{array}$ & $m / z$ & Related pathway \\
\hline$\overline{E S I}(+)$ & Stearoylcarnitine & $(\mathrm{M}+\mathrm{H})+$ & 1.5381 & 1.8064 & 0.0347 & 254.1865 & 428.3754 & Fatty acid beta-oxidation \\
\hline ESI $(+)$ & L-Palmitoylcarnitine & $(\mathrm{M}+\mathrm{H})+$ & 1.4626 & 1.6676 & 0.0158 & 263.4055 & 400.3447 & Fatty acid beta-oxidation \\
\hline ESI (+) & Cholic acid & $\begin{array}{l}(\mathrm{M}+ \\
\left.\mathrm{NH}_{4}\right)+\end{array}$ & 10.7858 & 3.6272 & 0.0358 & 383.6785 & 426.3243 & Primary bile acid biosynthesis \\
\hline ESI (-) & Taurine & $(\mathrm{M}-\mathrm{H})-$ & 1.1519 & 1.3343 & 0.0687 & 537.531 & 124.0072 & Primary bile acid biosynthesis \\
\hline ESI $(+)$ & Adenosine & $\begin{array}{l}(\mathrm{M}+\mathrm{H}- \\
\left.\mathrm{H}_{2} \mathrm{O}\right)+\end{array}$ & 1.2463 & 2.4131 & 0.0919 & 163.891 & 250.0973 & Purine metabolism \\
\hline ESI (-) & Hypoxanthine (HYPO) & $(\mathrm{M}-\mathrm{H})-$ & 4.6457 & 0.4923 & 0.0350 & 304.1335 & 135.0318 & Purine metabolism \\
\hline ESI (-) & L-Tryptophan & $(\mathrm{M}-\mathrm{H})-$ & 1.1472 & 0.8695 & 0.0608 & 469.231 & 203.0821 & Biosynthesis of proteins \\
\hline ESI $(-)$ & D-Mannose & $\begin{array}{l}(\mathrm{M}+ \\
\left.\mathrm{CH}_{3} \mathrm{COO}\right)-\end{array}$ & 1.3052 & 0.7540 & 0.0746 & 560.7445 & 239.0763 & Antioxidation \\
\hline ESI (-) & 4-Pyridoxic acid & $(\mathrm{M}-\mathrm{H})-$ & 3.0237 & 0.5923 & 0.0002 & 69.6135 & 182.0455 & Vitamin B6 metabolism \\
\hline ESI (+) & 4-Pyridoxic acid & $(M+H)+$ & 2.9734 & 0.5609 & 0.0001 & 67.7175 & 184.0648 & Vitamin B6 metabolism \\
\hline $\mathrm{ESI}(+)$ & $\begin{array}{l}\text { 1-Stearoyl-sn-glycerol 3- } \\
\text { Phosphocholine (LPC) }\end{array}$ & $\begin{array}{l}(\mathrm{M}-\mathrm{H}+ \\
2 \mathrm{Na})+\end{array}$ & 1.4861 & 1.4210 & 0.0037 & 283.888 & 568.3406 & $\begin{array}{l}\text { Major phospholipid components } \\
\text { in serum (liver toxicity) }\end{array}$ \\
\hline ESI (+) & $\begin{array}{l}\text { 1,2-Di-(9Z-octadecenoyl) } \\
\text {-sn-glycero-3-phosphocholine } \\
\text { (DOPC) }\end{array}$ & $(M+H)+$ & 11.7198 & 0.5475 & 0.0848 & 169.062 & 786.5999 & Structure of cell membranes \\
\hline ESI (+) & Acetylcarnitine & $(M+H)+$ & 3.1389 & 0.8104 & 0.0811 & 564.452 & 204.1271 & $\begin{array}{l}\text { Related to ammonia } \\
\text { concentration }\end{array}$ \\
\hline ESI $(-)$ & Salicyluric acid & $(\mathrm{M}-\mathrm{H})-$ & 2.9182 & 0.3989 & 0.0000 & 302.264 & 194.0461 & Energy metabolism \\
\hline ESI $(-)$ & Salicylic acid & $(\mathrm{M}-\mathrm{H})_{-}^{-}$ & 7.7978 & 0.3045 & 0.0001 & 64.863 & 137.0247 & Immune function modulation \\
\hline ESI (-) & DL-Lactate & $(\mathrm{M}-\mathrm{H})-$ & 11.3476 & 0.7210 & 0.0316 & 413.5235 & 89.02506 & $\begin{array}{l}\text { Central carbon metabolism in } \\
\text { cancer }\end{array}$ \\
\hline
\end{tabular}

oxidation that function as chaperones for fatty-acyl CoA transport into the mitochondria [12], can enhance energy and physical function as well as play a major role in the transfer and use of lipids [13]. Here, a higher level of stearoylcarnitine and L-palmitoylcarnitine was observed in the OG sheep than in the LG sheep, which reflected the elevated fatty acid metabolism in the former [14, 15]. This result was consistent with the lower serum levels of NEFAs in the present study and our previously obtained transcriptomic data (unpublished data). We found that
A

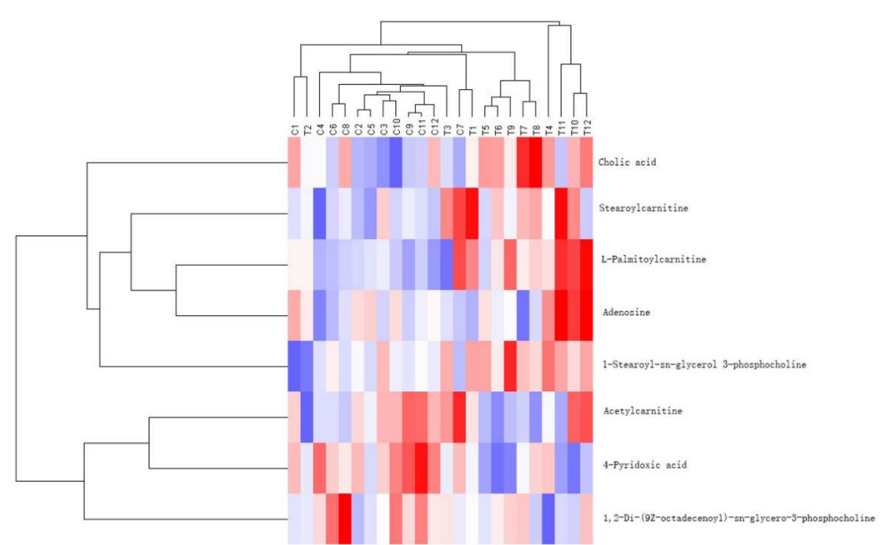

B

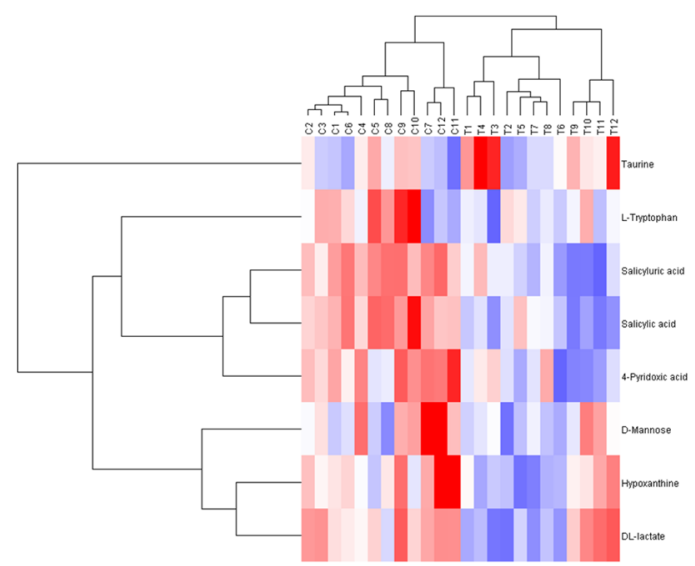

Fig. 4 Unsupervised hierarchical clustering heat map of metabolites obtained from the serum of sheep in the positive mode (a) and negative mode (b) 
several differentially expressed hepatic genes related to the peroxisome proliferator-activated receptor (PPAR) pathway and fatty acid oxidation were significantly upregulated in the OG sheep. It was reported that additional energy utilized for animal physical activity would be necessary under increased grazing intensity, which would then reduce the available energy required for growth and production [16]. Therefore, the metabolomic and transcriptomic profiles described above may partially explain the reduced growth performance of sheep under OG conditions.

Bile acids (BAs) are a group of structurally similar compounds that function in lipid metabolism as well as intestinal signaling [17]. In addition, individual BA analysis may provide valuable information relating to the development of toxic liver injury and disease [18]. Cholic acid is one of the two major BAs produced by the liver [19]. The elevation of cholic acid is a potential biomarker of liver injury in various in vivo experimental models, and extra cholic acid may partially contribute to an increased risk of inflammation [20]. Taurine is involved in the primary BA biosynthesis pathway and conjugates with BAs to form bile salts, which are necessary for proper lipid absorption during digestion [21]. Higher levels of taurine have also been reported in several types of disease due to apoptosis [22-24]. Previous studies demonstrated that liver and gastrointestinal dysfunction disturbed hepatic synthesis and BA clearance, thereby resulting in alternations in serum BA profiles [25]. In the present study, classical biomarkers of hepatotoxicity, such as ALT, AST, ALP and TBIL, were significantly elevated in the OG group. The reason for this phenomenon may be the imbalanced nutritional contents of the herbage, especially higher CP and lower NFE contents in the herbage under OG conditions, which was also observed in our previous study [4]. Therefore, the increased levels of cholic acid and taurine suggest that OG may induce liver injury in sheep. However, it is reported that AST and ALT are not specific marker of liver damage in sheep [26]. Thus, direct evidence, such as hepatic tissue HE staining and immunohistochemical staining, is still needed, and will be performed in an additional study in the future.

Purine nucleosides participate in multiple intracellular neuronal processes and provide DNA and RNA precursors [27]. The primary role of purine metabolism is in the antioxidant defense system during neurodevelopment $[28,29]$. Adenosine is a signaling nucleoside that is produced following tissue injury [30]. The production of extracellular adenosine and its subsequent signaling through adenosine receptors play an important role in orchestrating injury responses in a variety of organs [30]. Hypoxanthine is one of the end products in the catabolism of adenosine [31]. Lower levels of hypoxanthine can be considered a sign of less degradation of adenosine, indicating higher concentrations of adenosine in biofluids in the body [32]. In sleep loss or sleep deprivation experimental model groups, serum adenosine was elevated, but its product of degradation, hypoxanthine, was decreased, suggesting disordered nucleotide metabolism [33]. It was reported that with increasing grazing intensity, sheep decreased their time at rest to maintain their grazing time [16]. In the present study, an increase in the concentration of adenosine and a reduced level of hypoxanthine were observed in sheep serum under OG conditions, indicating that a lack of resting time due to OG might induce a disturbance of nucleotide metabolism in sheep.

A reduced level of L-tryptophan (Trp) was observed in the OG group in the current study. Trp is an important branched chain amino acid that is considered as a building block in protein synthesis as well as a vital biochemical precursor in multiple neurofunctional compounds [34]. It was recently reported that Trp was the third most limiting amino acid in growing lambs [35]. In a separate project, our laboratory detected differences in hepatic proteomics profiles between OG and LG groups [4]. Briefly, our observations demonstrated that a Trp catabolism enzyme (kynureninase, KYNU) [36] was more abundant in the hepatic proteome of OG sheep than in that of LG sheep. Thus, in the current study decreased levels of Trp under OG conditions reflect higher proteolysis or the impairment of protein synthesis, which is consistent with the lower concentration of serum TP in the OG group. Furthermore, lower levels of acetylcarnitine were observed in the OG group. Acetylcarnitine performs major functions as a cofactor for the transport of long-chain fatty acids through the mitochondrial membrane [37]. However, previous research has demonstrated that acetylcarnitine deficiency leads to increased ammonia levels in the body [38]. Our former research has also proved that the expression a hepatic protein (histidine ammonia-lyase, HAL) involved in ammonia elimination was increased in sheep under OG conditions [4]. Thus, above metabolomics and proteomics data could be a reason for the increased concentration of BUN in the OG group. Altered levels of stearoylcarnitine, L-palmitoylcarnitine, Trp and acetylcarnitine combined with the results of serum levels of NEFA, GLU and BUN indicate that sheep switch energy sources from carbohydrates to proteins and fatty acids under the OG condition, causing poorer nitrogen utilization efficiency and extra lipolysis. For energy metabolism, a number of studies revealed lower plasma GLU and ALB, and higher urea levels of ewes under restricted nutritional conditions [39-41]. This phenomenon was consistent with up-regulated expression of KYNU and HAL in our previously obtained hepatic proteomics data [4]. Thus, we speculate that Trp might be an important biomarker of OG, which could also 
explain the reduced sheep growth performance. In accordance with the Trp level, 4-pyridoxic acid and salicylic acid were reduced in OG sheep in the current study. Lower levels of 4-pyridoxic acid reflect vitamin B6 dysfunction [42]. The active form of vitamin B6 is involved in various enzyme reactions that are related to amino acid and lipid metabolism [43]. In contrast, defects in vitamin B6 affected antioxidant defenses in animal livers [44, 45]. Salicylic acid is a 5-aminosalycilic acid precursor and suppresses IL- 8 secretion by TNF- $\alpha$-activated intestinal epithelial cells [46]. In this study, increased concentration of IL-8 and reduction of 4-pyridoxic, salicylic acid and IgG were observed simultaneously in the OG group, which was consistent with the above research conclusion that sheep may be under environmental stresses due to the induction of certain inflammatory factors [47] and inadequate disease resistance under OG conditions. Previous research demonstrated that serum IgG concentration had significant linear association with buffalo calves average daily gain (ADG) from birth to day 30 [48]. Therefore, the lower levels of 4-pyridoxic, salicylic acid and IgG integrated with higher level of IL-8 in the present study may be induced by the reduction of sheep growth performance under OG conditions. Our previous hepatic proteomics data showed that the expression of proteins involved in immune and inflammatory responses were downregulated and upregulated, respectively, in the OG sheep [4], which was consistent with the metabolomic data obtained in the present study. However, it is important to note that this study reported immune and inflammatory responses in whole blood that does not entirely indicate functional changes. Thus, these results should be interpreted with caution and further research study is needed to confirm the effect of OG on innate and adaptive immunity using specific immune cells such as neutrophils, rather than whole blood [47].

\section{Conclusions}

In conclusion, sheep under the OG condition exhibited significantly reduced growth performance and evident changes of serum biochemical variables compared to sheep under the LG condition. Simultaneous changes of serum metabolites have also been identified by untargeted metabolomics using UHPLC-Q-TOF/MS, thereby providing a unique perspective concerning OG-triggered changes in cellular metabolites. Changes in 15 serum metabolites (6 metabolites exhibited a significant increase in concentration, and 9 metabolites showed a significant reduction in concentration) were identified in sheep under the OG condition and were mainly involved in fatty acid oxidation, bile acid biosynthesis and purine and protein metabolism. The identification of the targets of these metabolites improves our understanding of the molecular mechanism of reduced sheep growth performance under OG conditions.

\section{Methods}

\section{Animals and experimental design}

The present study was conducted following the Regulations for the Administration of Affairs Concerning Experimental Animals of the State Council of the People's Republic of China. The Committee on Experimental Animal Management of the Chinese Academy of Agricultural Sciences, (Beijing), approved our research protocol (Approval No. 46/28.05.2016).

We conducted this research at the Institute of Grassland Research of the Chinese Academy of Agricultural Sciences Research Station in 2016 during the summer. The site is located at the Xilin River basin in the Inner Mongolia Autonomous Region of China (116 $32^{\prime}$ E, $44^{\circ} 15^{\prime} \mathrm{N}$ ). Its predominant natural vegetation included three species of grass: Leymus chinensis, Stipa krylovii, and $S$. grandis.

In June 2016, a total of 48 Uzhumchin wethers (average age: 24 months; baseline live weight (LW):33.2 $\pm 3.9 \mathrm{~kg}$ ) were obtained from the Xinshengjia Sheep Breeding Company (Xilinhot, Inner Mongolia, China). We randomly assigned the wethers to either the LG (4 sheep/plot) or the OG (12 sheep/plot) groups. The experimental site comprised six plots $(1.33 \mathrm{ha} / \mathrm{plot}$, three plots per grazing group). Two different stocking rates were used, namely, 3.0 (LG) and 9.0 sheep/ha (OG) (Fig. 5). The wethers were allowed to graze without interruption in the plots during

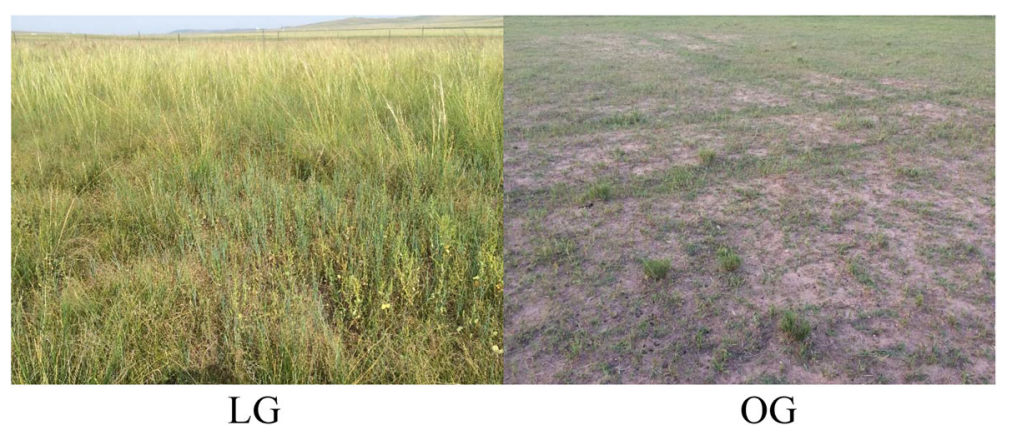

Fig. 5 Grazing differences between LG plots and OG plots 
the duration of the experiment (June 5 to September 3, 90 days). At the start of the research study, sheep were treated with an anthelmintic to eliminate the infection of internal parasites [2] and given water and mineral lick stones ad libitum. After the grazing experiment, all sheep were released.

\section{Sample collection}

To identify the chemical constituents of the sheep diet, we collected plants every month of the grazing experiment. The protocol for plant collection was as described elsewhere [49] and the details are provided as supplementary material (see Additional file 2). The plants were assessed for different chemical composition indices such as CP, gross energy, NFE, neutral detergent fiber (NDF), acid detergent fiber (ADF), as well as ADL, as described elsewhere (a single pooled sample per each plot, $n=3$ ) [2]. The LW of the animals was identified on the study's first and the last day (after $12 \mathrm{~h}$ of fasting) and used it to calculate the mean daily gain. Blood was then collected from four sheep selected at random from each plot in the groups ( $n=12$ for each group) via venipuncture of the jugular vein and into vacuum tubes. The blood was then centrifuged twice at $2000 \mathrm{~g}$ at $4{ }^{\circ} \mathrm{C}$ for $30 \mathrm{~min}$ and then at $400 \mathrm{~g}$ at $4{ }^{\circ} \mathrm{C}$ for $10 \mathrm{~min}$ to isolate the sera, which were then kept at $-80^{\circ} \mathrm{C}$ to await further analysis.

\section{Biochemical, immune response and inflammation analyses}

For biochemical, immune response and inflammatory variables of the serum, the concentration of ALT, AST, ALP, ALB, TBIL, BUN, GLU, and NEFA were measured using a fully automatic biochemistry analyzer (Hitachi 7020, Tokyo, Japan); IL-8 and IgG were determined using a corresponding diagnostic kit (Nanjing Jiancheng Bioengineering Institute, Nanjing, China) according to the instructions of the manufacturer.

\section{Sample preparation}

Each sample was slowly thawed at $4{ }^{\circ} \mathrm{C}$. One volume of plasma $(50 \mu \mathrm{L})$ was mixed with three volumes of cold mixtures of methanol/acetonitrile/ $\mathrm{H}_{2} \mathrm{O}(2: 2: 1, \mathrm{v} / \mathrm{v} / \mathrm{v})$ to precipitate proteins following adequate vortexing, incubation $\left(-20^{\circ} \mathrm{C}\right.$ for $\left.1 \mathrm{~h}\right)$ and centrifugation $(15 \mathrm{~min}$ at 13 , $000 \mathrm{rpm}$ and $4{ }^{\circ} \mathrm{C}$ ). The supernatant was collected, dried in a vacuum, redissolved in $50 \mu \mathrm{L}$ of acetonitrile/water $(1: 1, \mathrm{v} / \mathrm{v})$, and centrifuged at $14,000 \mathrm{rpm}$ for $15 \mathrm{~min}, 4^{\circ} \mathrm{C}$. A pooled QC sample was created by taking the same amount of each sample and mixing the amounts together. We employed QC samples to evaluate the system's stability and performance before sample loading and during the whole experimental process (every five samples).

\section{Metabolomics analysis}

We conducted sample analyses using an ultra-highperformance liquid chromatography (UHPLC) system (Agilent 1290 Infinity LC system, USA) coupled with a premium quadrupole time-of-flight mass spectrometer (QTOF MS) system (AB Sciex TripleTOF 6600, MA, USA). Hydrophilic interaction liquid chromatography (HILIC) separation of metabolite samples was performed on a reversed-phase $\mathrm{C}_{18}$ ACQUITY UPLC BEH column $(2.1 \mathrm{~mm} \times 100 \mathrm{~mm}, 1.7 \mu \mathrm{m}$; Waters, Ireland). In both the ESI positive and negative modes, the flow rate was $400 \mu \mathrm{L} / \mathrm{min}$, with solvent A composed of $25 \mathrm{mM}$ ammonium acetate $+25 \mathrm{mM}$ ammonium hydroxide and solvent $\mathrm{B}$ composed of acetonitrile. The gradient consisted of $85 \%$ B for $1 \mathrm{~min}$, a linear reduction to $65 \%$ B in 11 min, a reduction to $40 \% \mathrm{~B}$ in $0.1 \mathrm{~min}$, holding this concentration for $4 \mathrm{~min}$, and then an increase to $85 \% \mathrm{~B}$ in $0.1 \mathrm{~min}$, with a $5 \mathrm{~min}$ re-equilibration period.

MS was operated with an AB Sciex TripleTOF mass spectrometer equipped with heated ESI positive and negative modes (AB Sciex TripleTOF 6600). The conditions of the ESI source were the following: ion source gas 1 (gas 1), 60; ion source gas 2 (gas 2), 60; curtain gas (CUR), 30; source temperature, $600^{\circ} \mathrm{C}$, and ion spray voltage floating (ISVF), $\pm 5500 \mathrm{~V}$. For MS-only acquisition, the instrument used an $\mathrm{m} / \mathrm{z}$ range of $60-1000 \mathrm{Da}$, with an accumulation time for the TOF MS scan was set at $0.20 \mathrm{~s} / \mathrm{spectra}$. For auto MS/MS acquisition, the instrument was set to within an $\mathrm{m} / \mathrm{z}$ range of $25-1000$ $\mathrm{Da}$, with an accumulation time of $0.05 \mathrm{~s} / \mathrm{spectra}$. We conducted the product ion scan with informationdependent acquisition (IDA) at a mode of high sensitivity. Collision energy (CE) was set at $35 \mathrm{~V}$ and $\pm 15 \mathrm{eV}$. The declustering potential (DP) was at $\pm 60 \mathrm{~V}$.

\section{Data processing and analysis}

The raw MS data using an instrument-specific format (.wiff) were converted to the common data format (MzXML) with the ProteoWizard tool msconvert (version 3.0.10051) and then processed by the program XCMS for feature assessment, retention time correction, as well as alignment. Metabolite identification was performed based on the accurate mass ( $<25 \mathrm{ppm})$ and MS/ MS data that were matched to our standard database. In ion features that were extracted, the subsequent analysis included only the variables showing $>50 \%$ of the nonzero measurement values in one or more groups.

After normalization that used total peak intensity, we uploaded the processed data to SIMCA-P (version 14.1, Umetrics, Umea, Sweden) for multivariate data analysis, including PCA as well as OPLS-DA. Sevenfold cross-validation as well as response permutation testing were employed to assess model robustness. The VIP value of every variable in the OPLS-DA 
model was assessed to determine its the contribution to classification. Furthermore, metabolites with a VIP value $>1$ were assessed using the Student's $t$-test at the univariate level to determine their significance [the normality test of data was conducted using SPSS Statistics 17.0 (SPSS, Inc., USA)], and a $P$ value $<0.05$ was deemed as statistically significant.

\section{Supplementary information}

Supplementary information accompanies this paper at https://doi.org/10. 1186/s12917-019-2218-9.

Additional file 1: Table S1. Effect of overgrazing on primary nutritional indexes of herbage.

Additional file 2. The detailed description of forage sample collection.

\section{Abbreviations}

KEGG: Kyoto encyclopedia of genes and genomes; LG: Light grazing; OG: Overgrazing; OPLS-DA: Orthogonal partial least-squares discriminant anaIysis; PCA: Principal component analysis

\section{Acknowledgements}

We thank American Journal Experts for their linguistic assistance during the preparation of this manuscript. Shanghai Applied Protein Technology provided the technical support in untargeted metabolomics analysis.

\section{Authors' contributions}

WR and JZ developed and framed the research questions. JZ and WR analyzed metabolomics data. YD and YG contributed to serum biochemical analysis and subsequent data analysis. HG contributed to revise the entire manuscript. JZ and WR drafted the manuscript. All authors have read and approved the manuscript.

\section{Funding}

This work was funded by the National Natural Science Foundation of China (31872407), the Fundamental Research Funds for Central Nonprofit Research Institutes (1610332018008), the Chinese National Key Basic Research and Development Program (2014CB138804). The funders had no role in study design, data collection and analysis, decision to publish, or the manuscript preparation.

\section{Availability of data and materials}

The datasets used and/or analyzed during the current study are available from corresponding author on reasonable request.

\section{Ethics approval and consent to participate}

This study was conducted in strict accordance with the Regulations for the Administration of Affairs Concerning Experimental Animals of the State Council of the People's Republic of China. The protocol was approved by the Committee on Experimental Animal Management of the Chinese Academy of Agricultural Sciences.

\section{Consent for publication}

Not applicable.

\section{Competing interests}

The authors declare that they have no competing interests.

\section{Author details}

'Key Laboratory of Forage Grass, Ministry of Agriculture, Institute of Grassland Research, Chinese Academy of Agricultural Sciences, Hohhot 010010, Inner Mongolia, China. ${ }^{2}$ College of Animal Science and Technology, Jilin Agricultural University, Changchun, Jilin 130018, China. ${ }^{3}$ College of Life Sciences, Inner Mongolia Agricultural University, Hohhot 010019, Inner Mongolia, China. ${ }^{4}$ School of Ecology and Environment, Inner Mongolia University, Hohhot 010021, Inner Mongolia, China.
Received: 5 July 2019 Accepted: 19 December 2019

Published online: 26 December 2019

\section{References}

1. Zhang R, Zhang W, Zuo Z, Li R, Wu J, Gao Y. Inhibition effects of volatile organic compounds from Artemisia frigida Willd. on the pasture grass intake by lambs. Small Ruminant Res. 2014;121(2-3):248-54.

2. Glindemanna T, Wang C, Tas B, Schiborrab A, Gierusb M, Taubeb F, et al. Impact of grazing intensity on herbage intake, composition, and digestibility and on live weight gain of sheep on the inner Mongolian steppe. Livest Sci. 2009;124(1-3):142-7.

3. Dickhoefer U, Bösing BM, Hasler M, Hao J, Lin L, Müller K, et al. Animal responses to herbage allowance: forage intake and body weight gain of sheep grazing the inner Mongolian steppe-results of a six-year study. J Anim Sci. 2016;94(5):2059-71.

4. Ren W, Hou X, Wang Y, Badgery W, Li X, Ding Y, et al. Overgrazing induces alterations in the hepatic proteome of sheep (Ovis aries): an iTRAQ-based quantitative proteomic analysis. Proteome Sci. 2017;15:2.

5. Elolimy A, Alharthi A, Zeineldin M, Parys C, Helmbrecht A, Loor JJ. Supply of methionine during late-pregnancy alters fecal microbiota and metabolome in neonatal dairy calves without changes in daily feed intake. Front Microbiol. 2019;10:2159.

6. Luo ZZ, Shen LH, Jiang J, Huang YX, Bai LP, Yu SM, et al. Plasma metabolite changes in dairy cows during parturition identified using untargeted metabolomics. J Dairy Sci. 2019;102(5):4639-50.

7. Jawor P, Zabek A, Wojtowicz W, Król D, Stefaniak T, Młynarz P. Metabolomic studies as a tool for determining the post-mortem interval (PMI) in stillborn calves. BMC Vet Res. 2019;15(1):189.

8. Li J, Wijffels G, Yu Y, Nielsen LK, Niemeyer DO, Fisher AD, et al. Altered fatty acid metabolism in long duration road transport: an NMR-based metabonomics study in sheep. J Proteome Res. 2011;10(3):1073-87.

9. Zhang RY, Zhu WY, Jiang LS, Mao SY. Comparative metabolome analysis of ruminal changes in Holstein dairy cows fed low-or high-concentrate diets. Metabolomics. 2017;13:74.

10. Tian H, Wang W, Zheng N, Cheng J, Li S, Zhang Y, et al. Identification of diagnostic biomarkers and metabolic pathway shifts of heat-stressed lactating dairy cows. J Proteome. 2015;125:17-28.

11. Yang $X$, Shen $Y$, Liu N, Wilson GWT, Cobb AB, Zhang Y. Defoliation and arbuscular mycorrhizal fungi shape plant communities in overgrazed semiarid grasslands. Ecology. 2018;99(8):1847-56.

12. Saylor PJ, Karoly ED, Smith MR. Prospective study of changes in the metabolomic profiles of men during their first three months of androgen deprivation therapy for prostate cancer. Clin Cancer Res. 2012;18(13):3677-85.

13. Fingerhut $R$, Röschinger W, Muntau AC, Dame T, Kreischer J, Arnecke $R$, et al. Hepatic carnitine palmitoyltransferase I deficiency: acylcarnitine profiles in blood spots are highly specific. Clin Chem. 2001:47(10):1763-8

14. Söder J, Wernersson S, Dicksved J, Hagman R, Östman JR, Moazzami AA, et al. Indication of metabolic inflexibility to food intake in spontaneously overweight Labrador retriever dogs. BMC Vet Res. 2019;15(1):96.

15. Zhang Y, Zhang W, Fu X, Zhou F, Yu H, Na X. Transcriptomics and metabonomics analyses of maternal DEHP exposure on male offspring. Environ Sci Pollut Res Int. 2018:25(26):26322-9.

16. Lin L, Dickhoefer U, Müller K, Susenbeth A. Grazing behavior of sheep at different stocking rates in the inner Mongolian steppe, China. Appl Anim Behav Sci. 2011;129(1):36-42.

17. Xiang $X$, Han Y, Neuvonen M, Laitila J, Neuvonen PJ, Niemi M. High performance liquid chromatography-tandem mass spectrometry for the determination of bile acid concentrations in human plasma. J Chromatogr B Analyt Technol Biomed Life Sci. 2010;878(1):51-60.

18. Luo L, Schomaker S, Houle C, Aubrecht J, Colangelo JL. Evaluation of serum bile acid profiles as biomarkers of liver injury in rodents. Toxicol Sci. 2014; 137(1):12-25.

19. Suzuki Y, Kaneko R, Nomura M, Naito H, Kitamori K, Nakajima T, et al. Simple and rapid quantitation of 21 bile acids in rat serum and liver by UPLC-MSMS: effect of high fat diet on glycine conjugates of rat bile acids. Nagoya J Med Sci. 2013;75(1-2):57-71.

20. Shimada T, Nakanishi T, Toyama A, Yamauchi S, Kanzaki A, Fujiwake H, et al. Potential implications for monitoring serum bile acid profiles in circulation 
with serum proteome for carbon tetrachloride-induced liver injury/ regeneration model in mice. J Proteome Res. 2010;9(9):4490-500.

21. Germain A, Ruppert D, Levine SM, Hanson MR. Metabolic profiling of a myalgic encephalomyelitis/chronic fatigue syndrome discovery cohort reveals disturbances in fatty acid and lipid metabolism. Mol BioSyst. 2017; 13(2):371-9.

22. Battini S, Faitot F, Imperiale A, Cicek AE, Heimburger C, Averous G, et al. Metabolomics approaches in pancreatic adenocarcinoma: tumor metabolism profiling predicts clinical outcome of patients. BMC Med. 2017; 15(1):56.

23. Meng $L$, Reyes H, Palma J, Hernandez I, Ribalta J, Sjövall J. Effects of ursodeoxycholic acid on conjugated bile acids and progesterone metabolites in serum and urine of patients with intrahepatic cholestasis of pregnancy. J Hepatol. 1997;27(6):1029-40.

24. Thompson MB, Blair PC, Morris RW, Neptun DA, Deyo DF, Popp JA. Validation and application of a liquid-chromatographic/enzymatic assay for individual bile acids in the serum of rats. Clin Chem. 1987;33(10): 1856-62.

25. Burkard I, von Eckardstein A, Rentsch KM. Differentiated quantification of human bile acids in serum by high-performance liquid chromatographytandem mass spectrometry. J Chromatogr B Analyt Technol Biomed Life Sci. 2005;826(1-2):147-59.

26. Braun JP, Trumel C, Bézille P. Clinical biochemistry in sheep: a selected review. Small Ruminant Res. 2010;92(1-3):10-8.

27. Wang H, Liu Z, Wang S, Cui D, Zhang X, Liu Y, et al. UHPLC-Q-TOF/MS based plasma metabolomics reveals the metabolic perturbations by manganese exposure in rat models. Metallomics. 2017;9(2):192-203.

28. Lara DR, Souza DO. Schizophrenia: a purinergic hypothesis. Med Hypotheses. 2000;54(2):157-66.

29. Yao JK, Keshavan MS. Antioxidants, redox signaling, and pathophysiology in schizophrenia: an integrative view. Antioxid Redox Signal. 2011;15(7):2011-35

30. Karmouty-Quintana H, Xia Y, Blackburn MR. Adenosine signaling during acute and chronic disease states. J Mol Med (Berl). 2013:91(2):173-81.

31. Niklasson F, Hetta J, Degrell I. Hypoxanthine, xanthine, urate and creatinine concentration gradients in cerebrospinal fluid. Ups J Med Sci. 1988;93(3):225-32

32. Brunstein MG, Ghisolfi ES, Ramos FLP, Lara DR. A clinical trial of adjuvant allopurinol therapy for moderately refractory schizophrenia. J Clin Psychiatry. 2005:66(2):213-9.

33. Gou XJ, Cen F, Fan ZQ, Xu Y, Shen HY, Zhou MM. Serum and brain metabolomic variations reveal perturbation of sleep deprivation on rats and ameliorate effect of total ginsenoside treatment. Int J Genomics. 2017;2017:5179271.

34. Kim YK, Myint AM, Verkerk R, Scharpe S, Steinbusch H, Leonard B. Cytokine changes and tryptophan metabolites in medication-naïve and medicationfree schizophrenic patients. Neuropsychobiology. 2009;59(2):123-9.

35. Van E Nolte J, Löest CA, Ferreira AV, Waggoner JW, Mathis CP. Limiting amino acids for growing lambs fed a diet low in ruminally undegradable protein. J Anim Sci. 2008;86(10):2627-41.

36. Harden JL, Lewis SM, Lish SR, Suárez-Fariñas M, Gareau D, Lentini T, et al. The tryptophan metabolism enzyme L-kynureninase is a novel inflammatory factor in psoriasis and other inflammatory diseases. J Allergy Clin Immunol. 2016;137(6):1830-40

37. Calabrese V, Giuffrida Stella AM, Calvani M, Butterfield DA. Acetylcarnitine and cellular stress response: roles in nutritional redox homeostasis and regulation of longevity genes. J Nutr Biochem. 2006:17(2):73-88.

38. Maldonado C, Guevara N, Queijo C, González R, Fagiolino P, Vázquez M. Carnitine and/or acetylcarnitine deficiency as a cause of higher levels of ammonia. Biomed Res Int. 2016;2016:2920108.

39. Chowdhury SA, Ørskov ER. Implications of fasting on the energy metabolism and feed evaluation in ruminants. J Anim Feed Sci. 1994;3: 161-9.

40. Caldeira RM, Belo AT, Santos CC, Vazques MI, Portugal AV. The effect of body condition score on blood metabolites and hormonal profiles in ewes. Small Ruminant Res. 2007;68(3):233-41.

41. Caldeira RM, Belo AT, Santos CC, Vazques MI, Portugal AV. The effect of long-term feed restriction and over-nutrition on body condition score, blood metabolites and hormonal profiles in ewes. Small Ruminant Res. 2007;68(3):242-55.
42. Zhang CE, Niu M, Li Q, Zhao YL, Ma ZJ, Xiong Y, et al. Urine metabolomics study on the liver injury in rats induced by raw and processed Polygonum multiflorum integrated with pattern recognition and pathways analysis. J Ethnopharmacol. 2016;194:299-306.

43. Huq MD, Tsai NP, Lin YP, Higgins L, Wei LN. Vitamin B6 conjugation to nuclear corepressor RIP140 and its role in gene regulation. Nat Chem Biol. 2007;3(3):161-5.

44. Anand SS. 2005. Protective effect of vitamin B6 in chromium-induced oxidative stress in liver. J Appl Toxicol. 2005;25(5):440-3.

45. Cabrini L, Bergami R, Fiorentini D, Marchetti M, Landi L, Tolomelli B. Vitamin B6 deficiency affects antioxidant defences in rat liver and heart. Biochem Mol Biol Int. 1998;46(4):689-97.

46. Beale D, Konstantinos K, Palombo E. Microbial metabolomics: applications in clinical, environmental, and industrial microbiology. London: Springer; 2016. p. 49-82.

47. Adeyemi JA, Harmon DL, Compart DMP, Ogunade IM. Effects of a blend of Saccharomyces cerevisiae-based direct-fed microbial and fermentation products in the diet of newly weaned beef steers: growth performance, whole-blood immune gene expression, serum biochemistry, and plasma metabolome1. J Anim Sci. 2019:97(11):4657-67.

48. Mastellone V, Massimini G, Pero ME, Cortese L, Avallone L. Effects of passive transfer status on growth performance in Buffalo calves. Asian-Aust J Anim Sci. 2011;24(7):952-6.

49. Hao J, Dickhoefer U, Lin L, Müller K, Glindemann T, Schönbach P, et al, Effects of rotational and continuous grazing on herbage quality, feed intake and performance of sheep on a semi-arid grassland steppe. Arch Anim Nutr. 2013;67(1):62-76

\section{Publisher's Note}

Springer Nature remains neutral with regard to jurisdictional claims in published maps and institutional affiliations.
Ready to submit your research? Choose BMC and benefit from:

- fast, convenient online submission

- thorough peer review by experienced researchers in your field

- rapid publication on acceptance

- support for research data, including large and complex data types

- gold Open Access which fosters wider collaboration and increased citations

- maximum visibility for your research: over $100 \mathrm{M}$ website views per year

At $\mathrm{BMC}$, research is always in progress.

Learn more biomedcentral.com/submissions 